УДК 512.54

\title{
On Normal Closures of Involutions in the Group of Limited Permutations
}

Yuri S. Tarasov*

Institute of Mathematics and Computer Science Siberian Federal University Svobodny, 79, Krasnoyarsk, 660041

Russia

Received 10.03.2016, received in revised form 08.05.2016, accepted 20.06.2016

We study the group $G=\operatorname{Lim}(N)$ of limited permutations of a set $N$ of all natural numbers. Found the link between the dispersion subsets of a set $N$ and normal subgroups of $G$.

Keywords: group, limited permutation, dispersion set, normal subgroup, involution.

DOI: 10.17516/1997-1397-2016-9-3-393-400.

\section{Introduction}

Let $N$ be the set of all natural numbers, $Z$ be the set of all integers, $M$ is any of these sets. The $S(M)$ will denote the group of all permutations of the set $M$.

Definition 1. Permutation $g \in S(M)$ is called limited if

$$
w(g)=\max _{\alpha \in M}\left|\alpha-\alpha^{g}\right|<\infty .
$$

If $g, h$ are limited permutations, so the same is about the permutations $g^{-1}$ and $g h$, as $w\left(g^{-1}\right)=w(g), w(g h) \leqslant w(g)+w(h)$. Thus set

$$
\operatorname{Lim}(M)=\{x \mid x \in S(M), w(x)<\infty\}
$$

form a group, which is a natural extension of a locally finite group $\operatorname{Fin}(M)$ of all finitary permutations of the set $M$, i.e. such permutation $y \in S(M)$, for which the set $\left\{\alpha \mid \alpha \in M, \alpha^{y} \neq \alpha\right\}$ is finite.

In the work of N.M. Suchkov [1] an example of the mixed group $H=A B$ was first built, where $A, B$ is periodic (and even locally-finite) subgroups. Then in $[2,3]$ it was found that

$$
H=\langle g|g \in \operatorname{Lim}(Z),| g \mid<\infty\rangle
$$

any countable free group and Aleshin 2-group isomorphically embeddable into the group $H$ and

$$
\operatorname{Lim}(Z)=H \lambda\langle d\rangle
$$

where $d$-shift, $\alpha^{d}=\alpha+1$ for any $\alpha \in Z$.

*gigtorus@yandex.ru

(c) Siberian Federal University. All rights reserved 
In their work [4] N. M. Suchkov and N. G. Suchkova proved the factorization of the whole group $\operatorname{Lim}(N)$ by two locally-finite subgroups and it is shown that the group $\operatorname{Lim}(M)$ is generated by the permutations $x \in S(M)$, for which $w(x)=1$.

These generators are either involutions, in which decomposition into independent cycles only transpositions of the $(\alpha \alpha+1), \alpha \in M$ or $M=Z$ and $x \in\left\{d, d^{-1}\right\}$. The relation between groups $\operatorname{Lim}(N)$ and $\operatorname{Lim}(Z)$ is found in [5]. Assuming that permutations of the group $S(N)$ have identical influence on the set $Z \backslash N$, we get a natural embedding of $S(N)<S(Z)$. Denote by $t$ the involution of the groups $S(Z)$ for which $\alpha^{t}=-\alpha(\alpha \in Z)$. It is proved that

$$
H=F i n(Z)\left(\operatorname{Lim}(N) \times(\operatorname{Lim}(N))^{t}\right) .
$$

From this congruence it follows that in the study of normal structure of the group $\operatorname{Lim}(Z)$ is the defining description of normal subgroups of the group $\operatorname{Lim}(N)$.

The first result in this direction is obtained in [5]. To formulate it is necessary to provide some definitions being introduced in this work. Let

$$
L=\left\{\mu_{1}, \mu_{2}, \ldots, \mu_{n}, \ldots\right\}
$$

is an infinite subset of $N$, where $\mu_{1}<\mu_{2}<\cdots<\mu_{n}<\ldots ; m$ is a fixed natural number. By definition, elements of $\mu_{i}$ and $\mu_{j}$ are equivalent, if $i=j$, or when $i<j(j<i)$ all inequations are fulfilled $\mu_{k+1}-\mu_{k} \leqslant m ; i \leqslant k \leqslant j-1(j \leqslant k \leqslant i-1)$. It is easy to see that this relation is indeed an equivalence relation, therefore it induces a decomposition of the set $L$ into equivalence classes. This partition is called $m$-partition. Let $B_{m}(L)$ be the set of all equivalence classes of elements of the set $L$.

Definition 2. The set $L$ is called $m$-dispersion, if all classes of the set $B_{m}(L)$ are finite and are completely $m$-dispersion if

$$
c_{m}=\max _{A \in B_{m}(L)}|A|<\infty .
$$

The set $L$ is called (completely) dispersion, if it is (completely) $m$-dispersion for every natural $m$.

The example of completely dispersion set is the set $L$, for elements of which the following inequations are used

$$
\mu_{2}-\mu_{1}<\mu_{3}-\mu_{2}<\cdots<\mu_{n}-\mu_{n-1}<\mu_{n+1}-\mu_{n}<\ldots
$$

Let $\mu_{n}+1<\mu_{n+1}(n=1,2, \ldots)$ and

$$
a=\left(\mu_{1} \mu_{1}+1\right)\left(\mu_{2} \mu_{2}+1\right) \ldots\left(\mu_{n} \mu_{n}+1\right) \ldots
$$

is the decomposition of an involution $a$ into independent transpositions. The main result of [5] is the theorem according to which the normal closure of the involution of $a$ in the group $\operatorname{Lim}(N)$ if and only if locally finite, when $L$ is an completely dispersion set.

Three hypotheses about normal subgroups of the group $\operatorname{Lim}(N)$ are provided ibid.

In this article one of these hipotheses is proved; namely, the following theorem is the main result of the present paper.

Theorem 1. An involution a if and only if contained in a proper normal subgroup of the group $\operatorname{Lim}(N)$ when $L$ is a dispersion set. If $L$ is dispersion, but is not completely dispersion set, then $\left\langle a^{g} \mid g \in \operatorname{Lim}(N)\right\rangle$ is a mixed group.

All the designations used in this work are either discussed, or standard [6]. 


\section{Preliminary results}

Let $\gamma, \varepsilon$ be integers and $\gamma \leqslant \varepsilon$. Let us call the set

$$
U_{\gamma}^{\varepsilon}=\{\beta \mid \beta \in Z, \gamma \leqslant \beta \leqslant \varepsilon\}
$$

a segment of integers; $\gamma$ is the left end of the segment, $\varepsilon$ is the right. In particular, $U_{\gamma}^{\gamma}=\{\gamma\}$.

For each $m \in N, \alpha \in L$ let be

$$
V_{\alpha}^{m}=U_{\alpha-m}^{\alpha+m} \bigcap N, E_{m}=\bigcup_{\alpha \in L} V_{\alpha}^{m} .
$$

Lemma 1. If the set $L$ is dispersion, then the set $E_{m}$ is 1-dispersion for every natural $m$.

Proof. If the Lemma is wrong, then such integers $m$ and $\gamma$ will be found that will prove that 1-decomposition of the $E_{m}$ set contains an infinite class $U_{\gamma}=\{\beta \mid \beta \in N, \beta \geqslant \gamma\}$. Let $\mu_{i}>\gamma$, then the union $V_{\mu_{i}}^{m} \bigcup V_{\mu_{i+1}}^{m}$ includes a segment of integers with $\mu_{i}, \mu_{i+1}$ endpoints. Therefore, $2 m$ is a decomposition of the set $L$ contains an infinite equivalence class with representative $\mu_{i}$ We have come to a contradiction with the dispersion of the set $L$. The Lemma is proved.

For brevity let us define $G=\operatorname{Lim}(N)$ and for each of dispersion set of $L$ we define the subgroup $Q=Q(L)$. As to Lemma 1 each set $E_{m}$ is split into segments

$$
W_{m 1}, W_{m 2}, \ldots, W_{m n}, \ldots
$$

of integers and if $\beta_{m n}$ is the right end of the segment $W_{m n}$, and $\alpha_{m n+1}$ is the left end of the segment $W_{m n+1}$, then $\alpha_{m n+1}>\beta_{m n}+1(n=1,2, \ldots)$; each segment is $W_{m n}$ is included into some interval $W_{m+1 s}$. Let

$$
Q_{m}=\left\{x \mid x \in G ; W_{m n}^{x}=W_{m n}(n=1,2, \ldots) ; \beta^{x}=\beta\left(\beta \in N \backslash E_{m}\right)\right\} .
$$

Obviously, $Q_{m}$ is a subgroup of $G$ and $Q_{m} \leqslant Q_{m+1}, m=1,2, \ldots$. Let, finally,

$$
Q=Q(L)=\bigcup_{m \in N} Q_{m}
$$

Lemma 2. $Q$ is proper normal subgroupin the group $G$.

Proof. Let $1 \neq h \in Q ; g \in G$ and $w(g)=k$. From the definition of the group $Q$, it follows that there are such natural $m$ that the element $h$ contains the subgroup $Q_{m}$. We claim that $h^{g} \in Q_{t}$, where $t=m+k+1$. Indeed, consider the decomposition of permutation $h$ into independent cycles. Since $h$ leaves untouched the segments of $W_{m n}(n=1,2, \ldots)$ and acts identically on the integers which are not contained in these segments, then all the cycles are finite. If $x=\left(\gamma_{1} \ldots \gamma_{s}\right)$ is one of these cycles is $(s>1)$, then $\gamma_{1}, \ldots, \gamma_{s}$ are contained in some interval $W_{m n}$, which coincides with the union of several segments

$$
V_{\mu_{q}}^{m}, V_{\mu_{q+1}}^{m}, \ldots, V_{\mu_{e}}^{m}
$$

We fix any number of $\gamma_{i}$ of set $\left\{\gamma_{1}, \ldots, \gamma_{s}\right\}$. Then $\gamma_{i} \in V_{\mu_{j}}^{m}$ for some index $j, q \leqslant j \leqslant e$; and since $\gamma_{i}^{x}=\gamma_{i}^{g}$ and $\left|\gamma_{i}-\gamma_{i}^{g}\right| \leqslant w(g)=k$, then $\gamma_{i}^{g} \in V_{\mu_{j}}^{t}$. Next, segment $W_{m n}$ is part of the segment

$$
V_{\mu_{q}}^{t} \bigcup V_{\mu_{q+1}}^{t} \bigcup \ldots \bigcup V_{\mu_{e}}^{t}
$$


In turn, this segment is contained in some segment $W_{t d}, d \in N$. Therefore, all elements of the cycle $x^{g}=\left(\gamma_{1}^{g} \ldots \gamma_{s}^{g}\right)$ belong to $W_{t d}$, where due to the definition of the group $Q_{t}$ we deduce that $x^{g} \in Q_{t}$ and $h^{g} \in Q_{t}$. Thus, $Q$ is a normal subgroup of $G$. It remains to show that $Q$ is proper subgroup of $G$. In fact, in the course the corroboration we have seen that any permutation of the subgroup $Q$ is decomposed into finite independent cycles.

Therefore an infinite cycle

$$
y=(\ldots 2 n \ldots 4213 \ldots 2 n-1 \ldots)
$$

is not contained in $Q$, but since $w(y)=2$, then $y \in G$. Thus, $Q \neq G$. The Lemma is proved.

Lemma 3. If $z$ is involution and $f$ is a triple of the cycle of the alternating group $A_{4}, z z^{f} z^{f^{2}}=1$.

Proof. We have $A_{4}=(\langle z\rangle \times\langle u\rangle) \lambda\langle f\rangle$, where $|u|=2$. Thus $f$ transitively permutes the involution $z, u, z u$ whose product equals 1 . Therefore, the Lemma is correct.

In further calculations we will use the well-known and easily verifiable

Assertion 1. Let $g, h$ are permutation of some set. If

$$
g=\ldots\left(\ldots \alpha_{1} \alpha_{2} \ldots\right) \ldots
$$

is decomposition of $g$ into independent cycles, then

$$
g^{h}=h^{-1} g h=\ldots\left(\ldots \alpha_{1}^{h} \alpha_{2}^{h} \ldots\right) \ldots .
$$

Lemma 4. Let $y=\left(\varepsilon_{1} \varepsilon_{2}\right)\left(\varepsilon_{3} \varepsilon_{4}\right)\left(\varepsilon_{5} \varepsilon_{6}\right)$ is decomposition of the permutation $y$ into independent transpositions, $f=\left(\varepsilon_{3} \varepsilon_{4} \varepsilon_{5}\right)$. Then yy $y^{f} f^{f^{2}}=\left(\varepsilon_{1} \varepsilon_{2}\right)$.

Proof. The elements $z=\left(\varepsilon_{3} \varepsilon_{4}\right)\left(\varepsilon_{5} \varepsilon_{6}\right), f$ generate a group isomorphic to alternating grope $A_{4}$, all elements of which commute with the transposition $\left(\varepsilon_{1} \varepsilon_{2}\right)$. Therefore, in view of Lemma 3 we have

$$
\begin{gathered}
y=\left(\varepsilon_{1} \varepsilon_{2}\right) z, y^{f}=\left(\varepsilon_{1} \varepsilon_{2}\right) z^{f}, y^{f^{2}}=\left(\varepsilon_{1} \varepsilon_{2}\right) z^{f^{2}}, \\
y y^{f} y^{f^{2}}=\left(\varepsilon_{1} \varepsilon_{2}\right)^{3} z z^{f} z^{f^{2}}=\left(\varepsilon_{1} \varepsilon_{2}\right) .
\end{gathered}
$$

The Lemma is proved.

Lemma 5. Let

$$
c=\left(\beta_{1} \beta_{1}+1\right)\left(\beta_{2} \beta_{2}+1\right) \ldots\left(\beta_{n} \beta_{n}+1\right) \ldots
$$

is the decomposition of permutation $c$ of the group $G$ into independent transpositions If all of the inequalities are fulfilled

$$
6 \leqslant \beta_{n+1}-\beta_{n} \leqslant m(n=1,2, \ldots),
$$

where $m$ is some fixed natural number, then the normal closure of $B(c)=\left\langle c^{g} \mid g \in G\right\rangle$ the involution of $c$ in the group $G$ contains a group Fin $(N)$ of all finitary permutations of the set $N$.

Proof. Since the group Fin $(N)$ coincides with the normal closure of any of its transposition, in order to prove the Lemma it is enough to show that $B(c)$ contains a transposition $\left(\beta_{1} \beta_{1}+1\right)$ from the decomposition of permutation $c$. In fact, since $\beta_{n+1}-\beta_{n} \geqslant 6$ for all natural $n$, the transpositions of the permutation's decomposition

$$
l=\left(\beta_{1} \beta_{1}+2\right)\left(\beta_{1}+1 \beta_{1}+3\right)\left(\beta_{2} \beta_{2}+2\right)\left(\beta_{2}+1 \beta_{2}+3\right) \ldots\left(\beta_{n} \beta_{n}+2\right)\left(\beta_{n}+1 \beta_{n}+3\right) \ldots
$$


is independent, and in the process $w(l)=2$, in particular $l \in G$. Therefore, the group $B(c)$ contains an involution $c^{e}$. Using the Assertion 1 we get

$$
c_{1}=c^{e}=\left(\beta_{1}+2 \beta_{1}+3\right)\left(\beta_{2}+2 \beta_{2}+3\right) \ldots\left(\beta_{n}+2 \beta_{n}+3\right) \ldots .
$$

Now let

$$
s=\left(\beta_{1}+2 \beta_{2}\right)\left(\beta_{1}+3 \beta_{2}+1\right)\left(\beta_{2}+2 \beta_{3}\right)\left(\beta_{2}+3 \beta_{3}+1\right) \ldots\left(\beta_{n}+2 \beta_{n+1}\right)\left(\beta_{n}+3 \beta_{n+1}+1\right) \ldots .
$$

By condition of the Lemma $\beta_{n+1}-\beta_{n} \leqslant m(n=1,2, \ldots)$, therefore, $w(s)<m$. Thus, $c_{1}^{s} \in B(c)$ and we get

$$
c_{1}^{s}=\left(\beta_{2} \beta_{2}+1\right)\left(\beta_{3} \beta_{3}+1\right) \ldots\left(\beta_{n+1} \beta_{n+1}+1\right) \ldots .
$$

Thus, $c c_{1}^{s}=\left(\beta_{1} \beta_{1}+1\right)$ is a contained in $B(c)$. The Lemma is proved.

Assertion 2( [5], Lemma 7). Let $\left\{\alpha_{1}, \ldots, \alpha_{k}\right\}$ is a subset of set $N$ and $\alpha_{i}+1<\alpha_{i+1}$, $1 \leqslant i \leqslant k-1$. If

$$
b=\left(\alpha_{1} \alpha_{1}+1\right)\left(\alpha_{2} \alpha_{2}+1\right) \ldots\left(\alpha_{k-1} \alpha_{k-1}+1\right)\left(\alpha_{k} \alpha_{k}+1\right)
$$

is the decomposition involutive permutation $b \in \operatorname{Fin}(N)$ into independent of transpositions,

$$
u=\left(\alpha_{1} \alpha_{2} \ldots \alpha_{k-1} \alpha_{k} \alpha_{k}+1 \alpha_{k-1}+1 \ldots \alpha_{2}+1 \alpha_{1}+1\right)
$$

is cycle, then the permutation $b b^{u}$ has the order $k$.

\section{Proof of the Theorem 1}

Let us proceed to the direct corroboration of the theorem formulated at the end of the introduction. Initially, it will prove the first part.

Suppose that $L$ is a dispersion set. Then from the constrution in Section 1 of the group

$$
Q=Q(L)=\bigcup_{n} Q_{n}
$$

and Lemma 2 it follows that the involution $a$ belong to the subgroup of $Q_{1}$, which is contained in proper normal subgroup $Q$ of $G$.

Conversely, suppose that $a$ is contained in proper normal in $G=\operatorname{Lim}(N)$ subgroup. Obviously, is equivalent to, subgroup $B(a)=\left\langle a^{g} \mid g \in G\right\rangle$ is a proper subgroup of $G$. Suppose that the set $L$ is not dispersion. This means that there is such a natural integer $m_{0}$ that the set $B_{m_{0}}(L)$ contains an infinite class of $A$. Then if $\mu_{\gamma}$ is the minimal number of the set $A$, then from definition it follows that $\mu_{i+1}-\mu_{i} \leqslant m_{0}$ for all $i \geqslant \gamma$. Hence we deduce that $B_{m}(L)$ consists of a single class $\{L\}$, if $m>\max \left(m_{0}, \mu_{\gamma}\right)$. Fix $m_{1}>m$. Thus,

$$
\mu_{n+1}-\mu_{n} \leqslant m_{1}(n=1,2, \ldots) .
$$

Now let us prove that $B(a)=G$. Then we get a contradiction to our assumption $B(a) \neq G$ and the first part of the theorem will be proved. Firstly it should be noted, that in the group $B(a)$ we can find a permutation

$$
c=\left(\beta_{1} \beta_{1}+1\right)\left(\beta_{2} \beta_{2}+1\right) \ldots\left(\beta_{n} \beta_{n}+1\right) \ldots,
$$


that for some natural $m$ all the inequations are fulfilled

$$
6 \leqslant \beta_{n+1}-\beta_{n} \leqslant m(n=1,2, \ldots) .
$$

Indeed, we will split the transpositions from decomposition $a$ into triples:

$a=\left(\mu_{1} \mu_{1}+1\right)\left(\mu_{2} \mu_{2}+1\right)\left(\mu_{3} \mu_{3}+1\right), \ldots\left(\mu_{3 k+1} \mu_{3 k+1}+1\right)\left(\mu_{3 k+2} \mu_{3 k+2}+1\right)\left(\mu_{3 k+3} \mu_{3 k+3}+1\right) \ldots$

Let

$$
t=\left(\mu_{2} \mu_{2}+1 \mu_{3}\right) \ldots\left(\mu_{3 k+2} \mu_{3 k+2}+1 \mu_{3 k+3}\right) \ldots
$$

Since $\mu_{n+1}-\mu_{n} \leqslant m_{1}$, therefore $w(t) \leqslant m_{1}$, and it means that, $t \in G$. Therefore, if $c=a a^{t} a^{t^{2}}$, then $c \in B(a)$ and by Lemma 4

$$
c=\left(\mu_{1} \mu_{1}+1\right) \ldots\left(\mu_{3 k+1} \mu_{3 k+1}+1\right) \ldots
$$

Thus from the inequation $2 \leqslant \mu_{n+1}-\mu_{n} \leqslant m_{1}$ it easily implies that $6 \leqslant \mu_{3 k+4}-\mu_{3 k+1} \leqslant 3 m_{1}=$ $m$. Assuming $\beta_{1}=\mu_{1}, \beta_{2}=\mu_{4}, \ldots, \beta_{n}=\mu_{3 n-2}, \ldots$, we get that the permutation $c$ is the sought fore.

Let us note that the inclusion $c \in B(a)$ immediately implies that $B(c) \leqslant B(a)$, and therefore for the proof of the part 1 of the Theorem it is enough to establish the congruence $B(c)=G$. It was noted in the introduction that the group $G$ is generated by involutions, in decomposition into independent transpositions of which only transpositions of the $(\alpha \alpha+1)$ form take part. Since $\operatorname{Fin}(N)<B(c)$ by Lemma 5 , to prove the congruence $B(c)=G$ it is enough to show that if

$$
x=\left(\gamma_{1} \gamma_{1}+1\right) \ldots\left(\gamma_{n} \gamma_{n}+1\right) \ldots,
$$

where $\gamma_{n+1}>\gamma_{n}+1(n=1,2, \ldots)$, then $x \in B(c)$. Since $B(c)$ contains any finitary permutation, $x_{n}=\left(\gamma_{1} \gamma_{1}+1\right) \ldots\left(\gamma_{n} \gamma_{n}+1\right)$, then without loss of generality we can assume that $\gamma_{1}>\beta_{1}$.

Denote $L_{x}=\left\{\gamma_{n} \mid n \in N\right\}$ and consider the case when the inequations are fulfilled for the elements of this set

$$
\gamma_{n+1}-\gamma_{n}>5 m(n=1,2, \ldots) .
$$

Let us split the set of $N \backslash\left\{1,2, \ldots, \beta_{1}\right\}$ into the segments of the integers is

$$
\Delta_{1}=U_{\beta_{1}+1}^{\beta_{3}}, \ldots, \Delta_{n}=U_{\beta_{2} n+1}^{\beta_{2 n+1}}, \Delta_{n+1}=U_{\beta_{2 n+1}+1}^{\beta_{2 n+3}}, \ldots .
$$

In virtue of the inequation (1)

$$
\left|\Delta_{n}\right|=\beta_{2 n+1}-\beta_{2 n-1}=\left(\beta_{2 n+1}-\beta_{2 n}\right)+\left(\beta_{2 n}-\beta_{2 n-1)} \leqslant 2 m .\right.
$$

From inequations (2) and $\gamma_{1}>\beta_{1}$ this implies that

$$
L_{x} \subset \bigcup_{n \in N} \Delta_{n} ;
$$

the intersection of $\Delta_{n} \cap L_{x}$ for every $n$ is either empty or contains max one element; $\gamma_{i}, \gamma_{j}$ is not contained in the adjacent segments for every $i \neq j$. Thus, there is such a sequence $j_{1}, j_{2}, \ldots, j_{n}, \ldots$, that $j_{n+1}-j_{n}>1(n=1,2, \ldots)$ and

$$
\gamma_{1} \in \Delta_{j_{1}}, \gamma_{2} \in \Delta_{j_{2}}, \ldots, \gamma_{n} \in \Delta_{j_{n}}, \ldots
$$


Let us define the permutation $\psi \in S(N)$ as follows: for $n=1,2, \ldots$ assume

$$
\begin{gathered}
\gamma_{n}^{\psi}=\beta_{2 j_{n}}, \quad \beta_{2 j_{n}}^{\psi}=\gamma_{n}, \quad\left(\gamma_{n}+1\right)^{\psi}=\beta_{2 j_{n}}+1, \quad\left(\beta_{2 j_{n}}+1\right)^{\psi}=\gamma_{n}+1 ; \\
\gamma^{\psi}=\gamma, \text { if } \gamma \notin \bigcup_{n \in N}\left(\left\{\gamma_{n}, \gamma_{n}+1\right\} \cup\left\{\beta_{2 j_{n}}, \beta_{2 j_{n}}+1\right\}\right) .
\end{gathered}
$$

Since the elements $\gamma_{n}, \beta_{2 j_{n}}$ belong to the segment $\Delta_{n}$ and $\left|\Delta_{n}\right| \leqslant 2 m$, then $w(\psi)<2 m$, i.e. $\psi \in G$. As to Assertion 1 we have

$$
x^{\psi}=\left(\beta_{2 j_{1}} \beta_{2 j_{1}}+1\right) \ldots\left(\beta_{2 j_{n}} \beta_{2 j_{n}}+1\right) \ldots
$$

Now let

$$
\alpha_{1}, \alpha_{2}, \ldots, \alpha_{n}, \ldots
$$

be elements of the set $\left\{\beta_{1}, \ldots, \beta_{n}, \ldots\right\} \backslash\left\{\beta_{2 j_{1}}, \ldots, \beta_{2 j_{n}}, \ldots\right\}$, arranged in ascending order. From the above it follows that if $\alpha_{i}=\beta_{k}$, then $\alpha_{i+1}$ is element of the set $\left\{\beta_{k+1}, \beta_{k+2}\right\}$, and therefore $\alpha_{i+1}-\alpha_{i} \leqslant \beta_{k+2}-\beta_{k} \leqslant 2 m$. Here $i$ is any natural number, $k=k(i)$. It's easy to deduce that the permutation

$$
f=\left(\alpha_{1} \alpha_{2} \alpha_{2}+1\right) \ldots\left(\alpha_{2 n-1} \alpha_{2 n} \alpha_{2 n}+1\right) \ldots
$$

is an element of the group $G$. Applying Lemmas 3, 4 we get the congruence $c c^{f} c^{f^{2}}=x^{\psi}$ from which it immediately follows that $x \in B(c)$.

Let us finally prove, that this inclusion is done in general case (without additional assumptions that for the elements of a set $L_{x}$ inequations are fulfilled (2)). To do this, we fix any natural number $s>5 \mathrm{~m}$, and represent the permutation $x$ as compositions of

$$
x=x_{1} x_{2} \ldots x_{s},
$$

where

$$
x_{i}=\left(\gamma_{i} \gamma_{i}+1\right)\left(\gamma_{i+s} \gamma_{i+s}+1\right) \ldots\left(\gamma_{i+k s} \gamma_{i+k s}+1\right) \ldots,
$$

$1 \leqslant i \leqslant s$. From the definition of the permutation of $x$ it implies that if $L_{x_{i}}=\left\{\gamma_{i+k s} \mid k=\right.$ $1,2, \ldots\}$, then the adjacent elements of this set an inequation is fulfilled

$$
\gamma_{i+(k+1) s}-\gamma_{i+k s}>s>5 m
$$

which coincides with the inequation (2) for the neighbouring elements of the set $L_{x}$. But then by proved above, $x_{i} \in B(c), 1 \leqslant i \leqslant s$, and therefore $x \in B(c)$. The first part of the theorem is proved.

Let us prove the second part. Let $L$ be a dispersion, but not comletely dispersion set. We need to show that the normal closure of $B(a)$ of an involution $a$ of a group $G$ contains an element of infinite order. Indeed, in view of the definition for some natural number $r$ there are pairwise disjoint sets

$$
L_{n}=\left\{\mu_{\alpha_{n}}, \mu_{\alpha_{n}+1}, \ldots \mu_{\beta_{n}}\right\},
$$

$n=1,2, \ldots$ of $L$ that $\left|L_{n}\right|>n$ and $\mu_{i+1}-\mu_{i} \leqslant r\left(\alpha_{n} \leqslant i \leqslant \beta_{n}-1\right)$. Let us define the permutation of the $u$ set $N$ by its decomposition into independent cycles $u_{n}(n=1,2, \ldots)$. Let

$$
u_{n}=\left(\mu_{\alpha_{n}} \mu_{\alpha_{n}+1} \ldots \mu_{\beta_{n}} \mu_{\beta_{n}}+1 \mu_{\beta_{n}}+1 \mu_{\beta_{n}-1}+1 \ldots \mu_{\alpha_{n}+1}+1 \mu_{\alpha_{n}}+1\right) \text {. }
$$

Then $w(u) \leqslant r$, i.e. $u \in G$. According to Assertion 2 the element $a a^{u} \in B(a)$ is decomposed into independent cycles which lengths is unbounded, and therefore $\left|a a^{u}\right|=\infty$. The theorem is proved. 
In conclusion, let us put an example of be a dispersion, but not comletely dispersion set. Let

$$
\begin{gathered}
L_{1}=\{2,3\}, L_{2}=\{4,5,6\}, L_{3}=\{8,9,10,11\}, \ldots, L_{n}=\left\{2^{n}, 2^{n}+1, \ldots, 2^{n}+n\right\} \\
L=L_{1} \bigcup L_{2} \bigcup L_{3} \bigcup \ldots \bigcup L_{n} \bigcup \ldots
\end{gathered}
$$

If $2^{n}$ is the representative of the class $A=A(n, m) \in B_{m}(L)$, then from definition it follows that $A$ contains a set $L_{n}$ of the $(n+1)$-th element, and if $m<2^{n}-\left(2^{n-1}+n-1\right)$, then $A=L_{n}$. Hence we conclude that the set $L$ is a dispersion, but not comletely dispersion set.

\section{References}

[1] N.M.Suchkov, An example of a mixed group factorized by two periodic subgroups, Algebra i Logika, 23(1984), no. 5, 385-387 (in Russian).

[2] N.M.Suchkov, On subgroups of the product of locally finite groups, Algebra $i$ logika, 24(1985), no. 4, 408-413 (in Russian).

[3] N.M.Suchkov, On the group of limited permutations, Konstruktsii v algebre i logike, Tver', 1990, 84-89 (in Russian).

[4] N.M.Suchkov, N.G.Suchkova, On groups of limited permutations, Journal of Siberian Federal University. Mathematics and physics, 3(2010), no. 2, 262-266 (in Russian).

[5] N.M.Suchkov, N.G.Suchkova, Normal subgroups of limited permuta tion groups, Siberskie electronnnye matematich. izvestiya, 12(2015), 344-353 (in Russian).

[6] M.I.Kargapolov, Y.I.Merzlyakov, Fundamentals of the theory of groups, New York, SpringerVerlag, 1979.

\section{О нормальных замыканиях инволюций в группе ограниченных подстановок}

Юрий С. Тарасов

Институт математики и фундаментальной информатики Сибирский федеральный университет Свободный, 79, Красноярск, 660041

Россия

Изучается группа $G=\operatorname{Lim}(N)$ ограниченных подстановок множества $N$ всех натуральных чисел. Найдена связь между рассеянными подмножествами множества $N$ и собственными нормальными подгруппами группы $G$.

Ключевые слова: группа, ограниченные перестановки, рассеивание, нормальная подгруппа, инволючии. 